\title{
The International Code of Virus Classification and Nomenclature (ICVCN): proposal for text changes for improved differentiation of viral taxa and viruses
}

\author{
Jens H. Kuhn • Sheli R. Radoshitzky • \\ Sina Bavari $\cdot$ Peter B. Jahrling
}

Received: 7 December 2012/ Accepted: 12 December 2012/Published online: 16 February 2013

(C) Springer-Verlag (outside the USA) 2013

\begin{abstract}
The International Committee on Taxonomy of Viruses (ICTV) is responsible for the classification of viruses into taxa. Importantly, the ICTV is currently not responsible for the nomenclature of viruses or their subclassification into strains, lineages, or genotypes. ICTV rules for classification of viruses and nomenclature of taxa are laid out in a code, the International Code of Virus Classification and Nomenclature (ICVCN). The most recent version of the Code makes it difficult for the unfamiliar reader to distinguish between viruses and taxa, thereby often giving the impression that certain Rules apply to viruses. Here, Code text changes are proposed to address this problem.
\end{abstract}

Disclaimer The content of this publication does not necessarily reflect the views or policies of the US Department of Health and Human Services, the US Department of Defense, the US Department of the Army, or of the institutions and companies affiliated with the authors. JHK performed this work as an employee of Tunnell Consulting, Inc., a subcontractor to Battelle Memorial Institute under its prime contract with NIAID, under Contract No. HHSN272200200016I.

\section{J. H. Kuhn ( $ه)$ · P. B. Jahrling}

Integrated Research Facility at Fort Detrick (IRF-Frederick), Division of Clinical Research (DCR), National Institute of Allergy and Infectious Diseases (NIAID), National Institutes of Health (NIH), B-8200 Research Plaza, Fort Detrick,

Frederick, MD 21702, USA

e-mail: kuhnjens@mail.nih.gov

S. R. Radoshitzky $\cdot$ S. Bavari

United States Army Medical Research Institute of Infectious

Diseases, Fort Detrick, Frederick, MD, USA

\section{Introduction}

The International Union of Microbiological Societies (IUMS) tasks the International Committee on Taxonomy of Viruses (ICTV) to make decisions on matters of virus classification and taxon nomenclature [4]. Statutes govern ICTV activities [7]. According to those, virus classification into taxa and taxon nomenclature are subject to rules (the Rules) set out in an international code (the Code). The most recent version of this International Code of Virus Classification and Nomenclature (ICVCN) is available in the latest, 9th, ICTV Report, which was published in 2011 [8].

The ICVCN is organized in three major parts: Section 1 outlines the "Statutory basis for the International Committee on Taxonomy of Viruses (ICTV)". Section 2 describes the "Principles of nomenclature". Finally, section 3 outlines the "Rules of Classification and Nomenclature" [capitalization or lack thereof is identical to the headlines in the code] [8].

In contrast to the International Code of Zoological Nomenclature Code (ICZN Code; http://www.iczn.org), the International Code of Nomenclature of Bacteria (ICNB; http://www.ncbi.nlm.nih.gov/books/NBK8817/), and the International Code of Nomenclature for Algae, Fungi, and Plants (ICNAFP; http://www.iapttaxon.org/nomen/main. php), the ICVCN is still short and much less elaborate. This article is the second of a series of manuscripts (first: [6]) that outlines proposals and suggestions that could make the ICVCN a more precise instrument for virus taxonomy, while at the same time decreasing currently existing confusion among laboratory virologists regarding the use of the Rules put forth in the Code. In particular, this article addresses the ongoing confusion among laboratory virologists regarding the difference of taxa (concepts of the mind) and viruses (physical entities), an important 
difference that the ICTV and the Code maintains. The current Code addresses only the classification of viruses into taxa and the nomenclature of taxa, but not the nomenclature of viruses or their subclassification. Recently, an updated version of the Code was proposed and accepted by the ICTV Executive Committee (TaxoProp 2011.002a-uG.A.v8; http://www.talk.ictvonline.org/files/ proposals/taxonomy_proposals_general1/m/gen04/4418. aspx). This new Code does not address the specific problem of differentiating viruses from taxa systematically, but addresses other important issues. Since the ICTV Executive Committee-accepted new version of the Code has yet to be ratified (and because ratification is not guaranteed), proposals made here are in regard to both the valid current Code and the forthcoming new version that might supersede it.

\section{Proposals for ICVNC text changes for improved differentiation of taxa and viruses}

ICVCN Rule 3.3 states:

"The ICTV is not responsible for classification and nomenclature of virus taxa below the rank of species. The classification and naming of serotypes, genotypes, strains, variants and isolates of virus species is the responsibility of acknowledged international specialist groups" [8].

Rule 3.3 is arguably the most important rule of the Code, and also the Rule that causes the most confusion among laboratory virologists. According to this Rule, the ICTV is responsible only for the organization of orders, families, subfamilies, genera, and species (the taxon levels currently in use). Several manuscripts have been published outlining the difference between taxa, including species, and viruses [1-3, 5, 9-12]. The content of these very important contributions to virus taxonomy does not need to be repeated here. However, for the unfamiliar reader it is important to remember that taxa, such as species, are manmade concepts (ideas) and that viruses are actual physical entities - and that the ICTV currently maintains this distinction. Consequently, viruses are not taxa, but they are members of taxa. For instance, species are not viruses, but viruses are members of species. Understanding this distinction clarifies that "serotypes, genotypes, strains, variants and isolates of virus species" cannot exist, at least not if "serotypes, genotypes, strains, variants and isolates of virus species" are understood as groups of actual entities. Viruses are unfortunately not mentioned in Rule 3.3 at all. Furthermore, "classification" is the assignment of physical entities, viruses, to taxa, i.e., the ICTV is responsible for the classification of viruses.
Proposal: To change the text of Rule 3.3 to

"The ICTV is responsible for the classification of viruses into taxa and the nomenclature of taxa. The lowest taxon in virus classification as understood by the ICTV is the virus species. The ICTV is not responsible for the nomenclature of virus names or the subdivision of viruses into serotypes, genotypes, strains, variants or isolates".

This clearer differentiation between taxa and viruses should be extrapolated to other Rules of the Code.

Support for changing the text of Rule 1.2(ii) and Rule 1.2(iv)

ICVCN Rule 1.2,

"The Statutes define the objectives of the ICTV. These are: (i) to develop internationally agreed names for virus taxa, including species and subviral agents... (iv) to maintain an Index of virus names" [8],

has already been accepted by the ICTV Executive Committee to be changed to

"The Statutes define the objectives of the ICTV. These are: (i) to develop internationally agreed names for virus taxa... (iv) to maintain an index of agreed names of virus taxa" (TaxoProp 2011.002a-uG.A.v8)

because "subviral agents" are physical entities, which by definition are not taxa. "Taxa, including...subviral agents" is therefore incorrect.

Proposal: To change the text of Rule 2.1

ICVCN Rule 2.1,

"The essential principles of virus nomenclature are..." [8]

could be changed to

"The essential principles of virus taxon nomenclature are..."

(according to Rule 3.3, the ICTV is not responsible for the nomenclature of viruses).

Proposal: To change the text of Rule 2.2

ICVCN Rule 2.2,

"Nomenclature of viruses and subviral agents is independent of other biological nomenclature ..." [8], 
which already has been accepted by the ICTV Executive Committee to be changed to

"Nomenclature of viruses is independent of other biological nomenclature ..." (TaxoProp 2011.002auG.A.v8),

could further be changed to

"Nomenclature of virus taxa is independent of other biological nomenclature ..."

(according to Rule 3.3, the ICTV is not responsible for the nomenclature of viruses).

Proposal: To change the text of Rule 3.1

ICVCN Rule 3.1,

"Virus classification and nomenclature shall be international and shall be universally applied to all viruses" [8]

could be changed to

"Virus classification into taxa and nomenclature of taxa shall be international and shall be universally applied to all virus taxa"

(according to Rule 3.3, the ICTV is not responsible for the nomenclature of viruses).

Proposal: To change the text of Rule 3.2

\section{ICVCN Rule 3.2,}

"The universal virus classification system shall employ the hierarchical levels of Order, Family, Subfamily, Genus, and Species" [8]

could be changed to

"The universal virus classification system shall employ the hierarchical taxon levels of order, family, subfamily, genus, and species"

(insertion of the word "taxon" emphasizes the difference between viruses and taxa).

Proposal: To change the text of Rule 3.5

\section{ICVCN Rule 3.5,}

"Taxa will be established only when representative member viruses are sufficiently well characterized and described in the published literature so as to allow them to be identified unambiguously and the taxon to be distinguished from other, similar taxa" [8] needs to be clarified. For one, it is unclear what "sufficiently well characterized" means and it is probably debatable what accounts for "published literature" (peerreviewed; indexed in PubMed?). Furthermore, since taxa are concepts, they cannot be "identified unambiguously" - only their members can be. The grammatical structure "Taxa will be established only... so as to... and the taxon to be distinguished from other similar taxa" is grammatically awkward. The ICTV could change the text of this Rule to:

"Taxa will be established only after ICTV Study Groups have judged representative potential member viruses to be characterized sufficiently to differentiate them from other viruses classified in already established taxa".

Support for changing the text of Rule 3.9

ICVCN Rule 3.9,

"Existing names of taxa and viruses shall be retained whenever feasible" [8]

has already has been accepted by the ICTV Executive Committee to be changed to

"Existing names of taxa shall be retained whenever feasible" (TaxoProp 2011.002a-uG.A.v8)

(according to Rule 3.3, the ICTV is not responsible for the nomenclature of viruses).

Support for changing the text of Rule 3.10

\section{ICVCN Rule 3.10,}

"The rule of priority in naming taxa and viruses shall not be observed" [8]

has already has been accepted by the ICTV Executive Committee to be changed to

"The rule of priority in naming taxa shall not be observed" (TaxoProp 2011.002a-uG.A.v8)

(according to Rule 3.3, the ICTV is not responsible for the nomenclature of viruses).

Support for changing the text of Rule 3.13

ICVCN Rule 3.13,

"Subscripts, superscripts, hyphens, oblique bars and Greek letters may not be used in devising new names" [8]

has already has been accepted by the ICTV Executive Committee to be changed to 
"Subscripts, superscripts, hyphens, oblique bars and Greek letters may not be used in devising new taxon names" (TaxoProp 2011.002a-uG.A.v8)

(according to Rule 3.3, the ICTV is not responsible for the nomenclature of viruses).

Proposal: To change the text of Rule 3.18

ICVCN Rule 3.18,

"New names shall be selected such that they, or parts of them, do not convey a meaning for the taxon which would either (1) seem to exclude viruses which lack the character described by the name but which are members of the taxon being named, or (2) seem to exclude viruses which are as yet undescribed but which might belong to the taxon being named, or (3) appear to include within the taxon viruses which are members of different taxa" [8].

could be changed to

"New taxon names shall be selected such that they, or parts of them, do not convey a meaning for the taxon which would either (1) seem to exclude viruses which lack the character described by the taxon name but which are members of the taxon being named, or (2) seem to exclude viruses which are as yet undescribed but which might belong to the taxon being named, or (3) appear to include within the taxon viruses which are members of different taxa".

This change would remove ambiguity regarding "names".

Proposal: To change the text of Rule 3.19

ICVCN Rule 3.19,

"New names shall be chosen with due regard to national and/or local sensitivities. When names are universally used by virologists in published work, these or derivatives shall be the preferred basis for creating names, irrespective of national origin" [8].

could be changed to

"New taxon names shall be chosen with due regard to national and/or local sensitivities. When taxon names are universally used by virologists in published work, these or derivatives shall be the preferred basis for creating taxon names, irrespective of national origin".

This change would remove ambiguity regarding "names".
Proposal: To change the text of Rules 3.26, 3.29, 3.31, and 3.33

ICVCN Rule 3.26,

"A genus is a group of species sharing certain common characters" [8]

could be changed to

"A genus is a group of species whose members share certain common characters";

ICVCN Rule 3.29,

"A subfamily is a group of genera sharing certain common characters. The taxon shall be used only when it is needed to solve a complex hierarchical problem" [8]

could be changed to

"A subfamily is a group of genera whose members share certain common characters. A subfamily can include a single genus if this is necessary to solve a complex hierarchical problem";

ICVCN Rule 3.31,

"A family is a group of genera (whether or not these are organized into subfamilies) sharing certain common characters" [8]

could be changed to

"A family is a group of genera (whether or not these are organized into subfamilies) whose members share certain common characters. A family can include a single genus if this is necessary to solve a complex hierarchical problem";

ICVCN Rule 3.33,

"An order is a group of families sharing certain common characters" [8]

could be changed to

"An order is a group of families whose members share certain common characters. An order can include a single family if this is necessary to solve a complex hierarchical problem";

(taxa, such as genera, subfamilies, families or orders, are concepts of the mind and do not have characters [1-3, 5, 912]. Taxa should be devised only when they are needed to solve a complex hierarchical problem - since that is the reason why taxa are created in the first place. Finally, pointing out that a higher taxon can include only a single lower taxon is necessary as otherwise the ICTV is in violation of its own Rules. For instance, the ICTV accepted 
the species White bream virus to be solely included into the genus Bafinivirus (i.e., the genus Bafinivirus is not, at this time, a "group of species") and the genus Arenavirus to be solely included into the family Arenaviridae (i.e., the family Arenaviridae is not, at this time, a "group of genera"). Numerous other examples exist. It most likely would be easier for the ICTV to make the Code text changes suggested above than to abolish all single taxa included in higher rank taxa).

\section{Suggestions for text changes to ICVCN comments for improved differentiation of taxa and viruses}

The 9th ICTV Report contains comments below some of the ICVCN Rules to further elaborate on their correct interpretation. These comments often confuse, as the separation between taxa and viruses in the Code is not consistently applied. It is important to remember that these comments have been added to the Code but are not part of the Code, and that they have not been formally adopted. Changes to the Code require official proposals (which will be submitted once this article is in press), whereas changes to the comments do not, i.e. the changes below should be understood as suggestions rather than proposals.

The comment to ICVCN Rule 3.2,

“... Some families are classified together into Orders, but for many, the family is the highest level taxon in use...Contrasting examples of full classifications of some negative strand RNA viruses are: (1) species Mumps virus; genus Rubulavirus; subfamily Paramyxovirinae; family Paramyxoviridae; order Mononegavirales, and (2) species Rice stripe virus; genus Tenuivirus (see also Rule 3.41)"

could be changed to

“... Some families are included in orders, but often the family is the highest level taxon in use... Contrasting examples of full classifications of some negative strand RNA viruses are: (1) virus mumps virus; species Mumps virus; genus Rubulavirus; subfamily Paramyxovirinae; family Paramyxoviridae; order Mononegavirales, and (2) virus rice stripe virus; species Rice stripe virus; genus Tenuivirus (see also Rule 3.41)"

(lower taxa are included into higher taxa; the insertion of virus names emphasizes the difference between species and viruses).

The comment to ICVCN Rule 3.3:

"Particular virus isolates may be regarded as strains, variants, clusters or other subspecific entities that, together with other entities, constitute a species. Classification of such isolates is not the responsibility of the ICTV but is the responsibility of international specialty groups. It is the responsibility of ICTV Study Groups to decide if an isolate or a group of isolates should constitute a species. Deciding the names of serotypes, genotypes, strains, variants or isolates of virus species is not the responsibility of the ICTV. However, it is recommended that new names not be the same as, or closely similar to, names already in use (Rule 3.14 for taxa). When a particular virus isolate is designated to represent a species, the decision as to which name will be adopted for the species for formal taxonomic purposes will be the responsibility of the ICTV, usually based on recommendations of a particular Study Group working on behalf of the ICTV. The Study Group will be expected to consult widely so as to ensure the acceptability of names, subject to the Rules in the Code. The policy of the ICTV is that as far as is possible, decisions on questions of taxonomy and nomenclature should reflect the majority view of the appropriate virological constituency"

could be changed to

"Particular virus isolates may be regarded as strains, variants, clusters or other subspecific entities of a virus that is considered a member of a species. Classification of such isolates is not the responsibility of the ICTV but is the responsibility of international specialty groups, such as the ICTV Study Groups. It is the responsibility of ICTV Study Groups to decide if a virus and its isolates should be members of a species. Deciding the names of viruses and their serotypes, genotypes, strains, variants or isolates is not the responsibility of the ICTV. However, it is recommended that new taxon names (in italics) are not identical in spelling to the names of their virus members (not in italics) or closely similar to names already in use for other viruses or taxa. When a particular virus isolate is designated a member of a $\underline{\text { species, }}$, the decision as to which name will be adopted for the species for formal taxonomic purposes will be the responsibility of the ICTV, usually based on recommendations of a particular Study Group working on behalf of the ICTV. The Study Group will be expected to consult widely so as to ensure the acceptability of taxon names, subject to the Rules in the Code"

(the last sentence "The policy of the ICTV is that as far as is possible, decisions on questions of taxonomy and nomenclature should reflect the majority view of the 
appropriate virological constituency" could be deleted as there is no wide consensus among virologists regarding taxa. The fact that many virologists do not yet differentiate between taxa and viruses leads virologists of the constituency to protest proposed taxa (in particular species) names because they think that this means that virus names are being changed. The remaining text changes follow explanations elsewhere in this article).

The comment to ICVCN Rule 3.4,

"Naturally occurring isolates that have genomes formed from parts of the genomes of different strains of a virus, either by recombination between genome nucleic acids or by re-assortment of separate genome parts, will be classified either as species or subspecific entities in the same way that other isolates are classified. Neither artificial variants made by recombination or re-assortment nor mutant viruses are subject to the Rules in the Code"

could be changed to

"Naturally occurring isolates of a virus that have genomes formed from parts of the genomes of different variants of that virus or other viruses, evolved either by recombination between genome nucleic acids or by reassortment of separate genome parts, will be assigned to a species or subspecific entities in the same way that other isolates are classified. Neither artificial variants made by recombination or re-assortment nor mutant viruses are subject to the Rules in the Code"

(recombination or reassortment occurs among variants and strains of a virus. Strains are always variants, but variants are not necessarily strains, i.e., replacing the word "strain" with "variant" broadens the comment. Taxa are concepts, viruses and their isolates, variants, and strains are physical entities. "Naturally occurring isolates of a virus that have genomes formed from parts of the genomes of different variants of that virus, evolved either by recombination between genome nucleic acids or by re-assortment of separate genome parts, will be classified either as species..." is therefore wrong. Isolates will be classified as instances of a virus and that virus is already assigned to a species. If not, then the isolates may require the establishment of a new species).

The comment to ICVCN Rule 3.6,

"A species can be classified as an unassigned member of a family when no genus has been devised. For example, Suid herpesvirus 2 is a herpesvirus of vertebrates but is not a member of any of the currently recognized genera in the family Herpesviridae. Likewise, Groundnut rosette assistor virus resembles viruses in the family Luteoviridae but is not classified in any of the genera in that family. These viruses are each classified as an unassigned member of their respective families"

could be changed to

"A species in a family when no genus has been devised. For example, the species Suid herpesvirus 2 is a herpesvirus species whose members infect vertebrates but is not included into any of the currently recognized genera in the family Herpesviridae. Likewise, the member of the species Groundnut rosette assistor virus, groundnut rosette assistor virus, resembles virus members of the family Luteoviridae but is not classified in any of the genera in that family. These virus species whose members infect vertebrates are each classified as unassigned members of their respective families"

(taxa are not physical entities. Suid herpesvirus 2 is the name of a species. Suid herpesvirus 2 is the name of the virus member of that species. Therefore, the sentence "Suid herpesvirus 2 is a herpesvirus" is wrong. Likewise, "Groundnut rosette assistor virus" is a species and can therefore not "resemble" a virus. Lower rank taxa are not members of higher rank taxa, but they are included in higher-ranking taxa).

The comment to ICVCN Rule 3.7,

“...Proposal 1. Approve Beta gamma virus as a species containing strains known as "beta gamma virus" and "alpha beta virus".

Proposal 2. Create a genus to contain species resembling Beta gamma virus.

Proposal 3. Name the genus created by Proposal 2, Betavirus.

Proposal 4. Nominate Beta gamma virus as the type species of the genus Betavirus.

Proposal 5. Create a family to contain genus Betavirus and similar genera.

Proposal 6. Name the family created by Proposal 5 , Betaviridae.

Proposal 7. Assign species $\mathrm{X}, \mathrm{Y}$ and $\mathrm{Z}$ to genus Betavirus (such a proposal should include a listing of the parameters for discriminating between species in the genus Betavirus)".

could be changed to

“...Proposal 1. Approve Bega virus as a species for beta gamma virus and alpha beta virus.

Proposal 2. Create a genus to include the species Bega virus. 
Proposal 3. Name the genus created by Proposal 2 Betavirus.

Proposal 4. Nominate Bega virus as the type species of the genus Betavirus.

Proposal 5. Create a family to include the genus Betavirus.

Proposal 6. Name the family created by Proposal 5, Begamaviridae.

Proposal 7. Assign species $X, Y$ and $Z$ to the genus Betavirus (such a proposal should include a listing of the parameters for discriminating members of the different species in the genus Betavirus)".

- Spelling a species name the same way as one of its member viruses (e.g. creating a species Beta gamma virus for its member beta gamma virus) or spelling a genus name using the same word stem as used for a higher taxon (e.g. creating a genus name Betavirus and family name Betaviridae) is ill-advised as it may lead to confusion of taxa and virus members (if italicization rules are not followed diligently) or of members of one taxon with those of another (the members of both the genus Betavirus and the family Betaviridae would be called betaviruses), which is a violation of ICVCN Rules 2.1(ii), 3.14, 3.22, 3.23, and 3.24. Since sigla are explicitly allowed by the Code (ICVCN Rule 3.15), devising a new species name, such as Begavirus (for Beta gamma), or a new family name, such as Begamaviridae (for Beta gamma), is simple and straightforward

- The ICTV is not responsible for the nomenclature of viruses. There is currently no approval process for virus names. Consequently, novel virus names do not need to be placed in quotation marks even in the absence of established taxa for the novel viruses

- Species are not physical entities. They therefore cannot resemble anything.

- Species names are taxon names, and taxa names are to be italicized (" $X, Y$ and $Z$ ").

The comment to ICVCN Rule 3.8,

"A valid name is one that has been published, one that is associated with descriptive material, and one that is acceptable in that it conforms to the Rules in the Code. Accepted names will be kept in an "Index" by the ICTV"

could be changed to

"A valid taxon name is one that has been published, one that is associated with descriptive material, and one that is acceptable in that it conforms to the Rules in the Code. Accepted taxon names will be kept in an "Index" by the ICTV"

(this change will clarify that only taxon naming is addressed).

The comment to ICVCN Rule 3.9,

"A stable nomenclature is one of the principal aims of taxonomy and therefore changes to names that have been accepted will only be considered in exceptional circumstances, and then only because of serious conflict with the Rules"

could be changed to

"A stable nomenclature is one of the principal aims of taxonomy and therefore changes to names that have been accepted will only be considered when they are either in conflict with the ICVCN Rules or when the name change results in the removal of ambiguities or relieves confusion"

(taxonomy is always in flux, therefore always requiring name changes. These changes should be kept to a minimum if possible, but at the same time it should be easy for a Study Group to change the name of a taxon if, for example, it is not adopted by the larger virologist community.

The comment to ICVCN Rule 3.11,

"New taxon names shall not be made by adopting a person's name, by adding a formal ending to a person's name or by using part of a person's name to create a stem for a name. When existing names of species incorporate a person's name (for example, Mason-Pfizer monkey virus) continued usage of this name, in agreement with Rule 2.3 and 3.9 , is in general preferable to the creation of a new name"

could be changed to

"New taxon names shall not be created by adopting a person's name, by adding a formal ending to a person's name or by using part of a person's name to create a stem for a name. When existing names of species incorporate a person's name (for example, species Mason-Pfizer monkey virus) continued usage of this name is acceptable, but a name change would be preferred"

(laboratory virologists primarily work, describe, and search for viruses and rarely need to use taxon names. Changing existing taxon names to adhere more closely to recommendations of the ICVCN would not be problematic as long as the virus names would remain constant).

The comment to ICVCN Rule 3.14, 
"The name selected for a new taxon should not sound indistinguishable from the name of another taxon at any rank or from any taxon. For example, the existence of the genus Iridovirus means that forms of new name such as "irodovirus" or "iridivirus" are unacceptable as they are too easily confused with an approved name. Confusion can also be between species and genus names as both end in "virus". Thus, for example, the name selected for a genus typified by a species "Omega virus" would not be named "Omegavirus" because species and genus would then be too readily confused"

could be changed to

"The name selected for a new taxon should not sound indistinguishable from the name of another taxon at any rank or from any taxon. For example, the existence of the genus Iridovirus means that forms of new name such as "Irodovirus" or "Iridivirus" would be unacceptable as they are too easily confused with an approved name. Species and genus names can also be easily confused as both end in "virus". Thus, for example, the name selected for a genus typified by a species "Omega virus" should not be named "Omegavirus" because species and genus would then be too readily confused"

(taxa names are to be italicized according to the Rules). The comment to ICVCN Rule 3.23,

"Species names normally comprise more than one word. The styles used when virus names are devised differ according to the traditions of the particular fields of virology. For example, plant virus names are usually constructed as host + symptom + "virus" (e.g. tobacco necrosis virus) whereas, in contrast, viruses in the family Bunyaviridae are usually named after the location at which the virus was found + "virus" (e.g. Bunyamwera virus).

could be deleted, as this comment is most likely in regard to the creation of virus names, which does not fall under the purview of the ICTV. Alternatively, the comment could be changed to

"Species names normally comprise more than one word ("xyz" + "virus", e.g. Bunyamwera virus)".

The comment to ICVCN Rule 3.40,

"When used formally, as labels for taxonomic entities, the names Tobacco mosaic virus and Murray Valley encephalitis virus are in the correct form and typographical style. Examples of incorrect forms are Ustilago maydis virus $\mathrm{H}$ (not italic), Murray valley encephalitis virus (Valley is a proper noun) or tobacco mosaic virus (not capitalized or italic). Taxa are abstractions and thus when their names are used formally, these are written distinctively using italicization and capitalization. In other senses, such as an adjectival form (e.g. the tobacco mosaic virus polymerase) italics and capital initial letters are not needed. Equally, these are not needed when referring to physical entities such as virions (e.g. a preparation or a micrograph of tobacco mosaic virus). This Rule was introduced in 1998 and is in contradistinction to Rules in the Code published in the 6th ICTV Report"

could be changed to

"The species names Tobacco mosaic virus and Murray Valley encephalitis virus are in the correct form and typographical style. Examples of incorrect forms for species names are Ustilago maydis virus $\mathrm{H}$ (not italicized), Murray valley encephalitis virus (Valley is a proper noun and should be capitalized) or tobacco mosaic virus (not capitalized or italicized). Taxa are abstractions and thus when their names are used they are written distinctively using italicization and capitalization. Names of viruses, on the other hand, are not italicized and not capitalized (except if a component of the virus name is a proper noun). The names tobacco mosaic virus and Murray Valley encephalitis virus are in the correct form and typographical style. Adjectival forms (e.g. the tobacco mosaic virus polymerase) are consequently not written in italics and not capitalized because the adjective refers to the actual virus enzyme and not to a taxon"

(taxon names cannot be used informally and "adjectival forms" do not refer to taxa but to their members).

Comment placement

\section{Comment}

"Ratified changes will be published in Virology Division News in Archives of Virology, and in subsequent ICTV Reports",

currently placed below ICVCN Rule 1.3, could be moved below Rule 1.2(iii) ("The Statutes define the objectives of the ICTV. These are:...to communicate taxonomic decisions to the international community of virologists").

\section{Comment}

"See section 3.8"

currently placed under ICVCN Rule 2.5 could be deleted. 


\section{References}

1. Calisher CH, Mahy BWJ (2003) Taxonomy: get it right or leave it alone. Am J Trop Med Hyg 68:505-506

2. Calisher CH (2007) Taxonomy: what's in a name? Doesn't a rose by any other name smell as sweet? Croat Med J 48:268-270

3. Drebot MA, Henchal E, Hjelle B, LeDuc JW, Repik PM, Roehrig JT, Schmaljohn CS, Shope RE, Tesch RB, Weaver SC, Calisher $\mathrm{CH}$ (2002) Improved clarity of meaning from the use of both formal species names and common (vernacular) virus names in virological literature. Arch Virol 147:2465-2471

4. King AMQ, Adams MJ, Carstens EB, Lefkowitz EJ (eds) (2011) Virus taxonomy-ninth report of the International Committee on Taxonomy of Viruses. Elsevier, London

5. Kuhn JH, Jahrling PB (2010) Clarification and guidance on the proper usage of virus and virus species names. Arch Virol 155: 445-453

6. Kuhn JH, Radoshitzky SR, Bavari S, Jahrling PB (2013) The International Code of Virus Classification and Nomenclature (ICVCN): proposal to delete Rule 3.41. Arch Virol 158(1):297299
7. The International Committee on Taxonomy of Viruses (ICTV) (2011) The statutes of the ICTV. In: King AMQ, Adams MJ, Carstens EB, Lefkowitz EJ (eds) Virus taxonomy-ninth report of the International Committee on Taxonomy of Viruses. Elsevier, UK, pp 1269-1272

8. The International Committee on Taxonomy of Viruses (ICTV) (2011) The International Code of Virus Classification and Nomenclature. In: King AMQ, Adams MJ, Carstens EB, Lefkowitz EJ (eds) Virus taxonomy-ninth report of the International Committee on Taxonomy of Viruses. Elsevier, London, pp 12731277

9. van Regenmortel MHV (2003) Viruses are real, virus species are man-made taxonomic constructions. Arch Virol 148:2481-2488

10. van Regenmortel MHV (2006) Virologists, taxonomy and the demands of logic. Arch Virol 151:1251-1255

11. van Regenmortel MHV (2007) Virus species and virus identification: past and current controversies. Infect Genet Evol 7:133-144

12. van Regenmortel MHV (2009) Virus species. In: Mahy BWJ, van Regenmortel MHV (eds) Desk encyclopedia of general virology. Elsevier, Oxford, pp 37-41 